\title{
Prevention of adiposity by the oral administration of $\beta$-cryptoxanthin
}

\author{
Katsuhiko Takayanagi * \\ Research and Development Center, Unitika Ltd., Uji, Kyoto, Japan
}

\section{Edited by:}

Norio K. Ishida, National Institute of Advanced Industrial Science and

Technology, Japan

\section{Reviewed by:}

Timo Partonen, University of Helsinki, Finland

Norio K. Ishida, National Institute of Advanced Industrial Science and Technology, Japan

Mohammed Al-Abri, Sultan Qaboos University Hospital, Oman

${ }^{*}$ Correspondence:

Katsuhiko Takayanagi, Research and Development Center, Unitika Ltd., 23

Uji-Kozakura, Uji, Kyoto, Japan.

e-mail: kats-takayanagi@unitika.co.jp
$\beta$-Cryptoxanthin ( $\beta$-CRX) is a carotenoid found in human blood. It is specifically rich in Satsuma mandarin (Citrus unshiu Marc.) but very little in other fruits or vegetables. Several reports indicate the health promoting benefits of $\beta$-CRX. As we had reported visceral fat reduction on mildly obese male by the oral administration of $\beta-C R X$, a detailed mechanism has not been identified. To identify the mechanism, obese model mouse, TSOD was used in the present study. Oral administration of $\beta$-CRX repressed body weight, abdominal adipose tissue weight, and serum lipid concentrations on TSOD mice. The outstanding observation is the significant repression of adipocyte hypertrophy. DNA microarray analysis strongly indicates that the oral administration of $\beta$-CRX represses the inflammatory cytokine secretion and improves the lipid metabolism and the energy consumption. It also suggests these effects are partly mediated by PPAR- $\alpha$, not only lipid metabolism and adipocyte differentiation control but possibly internal circadian clock modulation.

Keywords: $\beta$-cryptoxanthin, Satsuma mandarin, TSOD, adipocyte, PPAR- $\alpha$, circadian control

\section{INTRODUCTION}

Nutritional importance of carotenoids is now widely accepted. They are known as natural antioxidants and anti-cancer effectors (Smith, 1998; Young and Lowe, 2001). In most cases carotenoid means $\beta$-carotene, lutein, or lycopene and the functional analysis of the other minor carotenoids are not fairly evaluated.

$\beta$-Cryptoxanthin ( $\beta$-CRX) is one of the carotenoids classified into xanthophyll and found in human blood together with $\alpha$-carotene, $\beta$-carotene, lycopene, lutein, and zeaxanthin. Unlike other abundant carotenoids, $\beta$-CRX is not found in most fruits or vegetables but in specific ones; hot pepper, persimmon, or Satsuma mandarin (Citrus unshiu Marc.; Mangels et al., 1993).

Satsuma mandarin, also known as table orange or Satsuma in western countries, is one of the most popular citrus in Japan. It is sweet, tasty, and rich in vitamin C. It is notable that Satsuma mandarin is one of the most $\beta$-CRX rich fruits in the world. An edible part of Satsuma mandarin contains about $1.8 \mathrm{mg} / 100 \mathrm{~g}$ of $\beta$-CRX, while it is $0.2 \mathrm{mg} / 100 \mathrm{~g}$ in Valencia orange and almost nothing in grapefruits. As $\beta$-CRX is rarely found in most fruits or vegetable, serum $\beta$-CRX concentration is almost parallel to the Satsuma mandarin consumption in Japanese population and is higher than western populations (Sugiura et al., 2002, 2004a).

As nutritional functions or metabolisms of abundant carotenoids, e.g., $\beta$-carotene or lycopene, are well studied (Kaplan et al., 1990; Cooney et al., 1991; Nair et al., 1996; Glise et al., 1998; Bramlev, 2000), those of $\beta$-CRX were not examined enough. Recent reports strongly suggest the important functions of $\beta$ CRX. They showed significant negative correlation between serum $\beta$-CRX concentrations and disease morbidity such as liver disorder (Sugiura et al., 2005, 2006a), cancer (Rauscher et al., 1998; Nishino et al., 2000; Tanaka et al., 2000), post-menopausal osteoporosis (Uchiyama and Yamaguchi, 2006; Sugiura et al., 2008, 2011), and diabetes (Sugiura et al., 2006b,c). These results strongly indicate $\beta$-CRX intake is beneficial for the human health.

Obesity is one of the most major symptoms of metabolic syndrome and the visceral fat accumulation causes many bad effects. Previous study suggests that $\beta$-CRX could normalize the serum lipid level (Sugiura et al., 2004b). We already showed the continuous $\beta$-CRX oral intake may improve the symptoms of metabolic syndrome, such as visceral adipose tissue, body weight, and waist circumference reduction in mildly obese males (Tsuchida et al., 2008). But we still do not know the mechanism how $\beta$-CRX would prevent obese and metabolic syndrome.

In this report, we investigated how $\beta$-CRX would prevent the symptoms of metabolic syndrome using obese model mouse, TSOD (Tsumura Suzuki obese diabetes; Hirayama et al., 1999; Suzuki et al., 1999), which accumulates visceral fat rapidly after 4 weeks old and shows similar symptoms of human metabolic syndrome.

\section{MATERIALS AND METHODS B-CRYPTOXANTHIN}

Enzyme processed Satsuma mandarin (EPSM) manufactured by UNITIKA Ltd. (Osaka, Japan) was used as the source of $\beta$-CRX. It is orange powder made from Satsuma mandarin pulp after juicing and contains minimum $0.2 \%(\mathrm{w} / \mathrm{w}) \beta$-CRX.

\section{TSOD AND TSNO MICE}

Male, 3 weeks old TSOD (Tsumura Suzuki obese, diabetes) and the genetic control (Tsumura Suzuki non-obese, diabetes, TSNO) mice were obtained from Institute for Animal Reproduction (Ibaraki, Japan). After a week acclimatization period, each mice 
were divided into Experimental groups and Control groups, denoted TSOD-E, TSOD-C, TSNO-E, and TSNO-C respectively ( $n=6$ in each). Olive oil-suspended EPSM, at $400 \mathrm{mg} / \mathrm{kg} /$ day, were orally administrated as $\beta$-CRX on Experimental groups (TSOD-E and TSNO-E), equivalent to $0.8 \mathrm{mg} \beta$-CRX/kg/day. Control groups (TSOD-C and TSNO-C) were administrated olive oil only.

Each group of mice were housed in one cage under $12 \mathrm{~h}$ light and $12 \mathrm{~h}$ dark cycle and fed Labo MR Stock (Nosan; Kanagawa, Japan). Body weight, food, and water consumption were monitored during the experimental period. The experimental period was 8 weeks. Three adipose tissues (epididymal, perirenal, and mesenteric adipose tissues), liver, femoral muscle, and serum were isolated after the experimental period.

This experiment was approved and carried out in accordance with the UNITIKA's Guidelines for Animal Experimentation, which are based on the notification of the Ministry of Education, Culture, Sports, Science, and Technology, Japan.

\section{SERUM LIPID ASSAY}

Three kinds of serum lipid concentrations, triglyceride (TG), total cholesterol (TC), and non-esterified fatty acid (NEFA) were analyzed using diagnosis kit (Wako; Osaka, Japan) following to the manufactures' instruction.

\section{ADIPOSE TISSUE ANALYSIS}

Epididymal adipose tissues were fixed in formalin, paraffin embedded and sectioned, following hematoxylin-eosin (HE) staining. The area of adipocytes was analyzed under microscope and Image Pro Discovery computer-assisted analysis system (Media Cybernetics, Inc.; Bethesda, MD, USA).

\section{DNA MICROARRAY ANALYSIS}

Isolated epididymal adipose tissue, liver, and femoral muscle were quickly placed in RNAlater (Ambion Inc., TX, USA) and kept in deep freezer $\left(-80^{\circ} \mathrm{C}\right)$ until needed. Total RNA was isolated from those tissues using RNeasy kit (QIAGEN, Tokyo, Japan) according to the manufacture's manual. Biotin-labeled cRNA was synthesized and fragmented using GeneChip 3'-IVT labeling kit (Affymetrix, Tokyo, Japan). Fragmented cRNA was hybridized at $45^{\circ} \mathrm{C}$ for $16 \mathrm{~h}$ to a GeneChip Mouse Genome 430A 2.0 Array (Affymetrix). Gene chips were washed and stained using fluid station and scanned using GeneChip scanner 300 7G (Affymetrix). Data were analyzed by Expression Console (Affymetrix) and imported into ArrayStar software (DNAstar). For biological interpretation of the differentially expressed genes, the database for annotation, visualization, and integrated discovery (DAVID) v6.2 was used (Huang et al., 2009) ${ }^{1}$. GO biological processes with $p<0.01$ were considered to be significantly changed. Differentially expressed genes were also categorized and mapped onto pathway using KEGG tools (Kanehisa, 1997).

\section{STATISTICAL ANALYSIS}

All data are shown as mean \pm SD. Data were analyzed using student T test and $p$ values $<0.05$ were considered statistically significant.

\footnotetext{
${ }^{1}$ http://david.abcc.ncifcrf.gov/
}

${ }^{2} \mathrm{http}: / /$ www.genome.jp/kegg/

\section{RESULTS}

\section{BODY WEIGHT}

The body weights on each group during the experimental period are shown in Figure 1. There was no difference on body weight between TSNO-E and TSNO-C. The average weight of TSOD-C is significantly higher than TSNO-C, because of the difference of genetic background.

The body weight of TSOD-E was significantly repressed compared to TSOD-C. It was statistically significant after the second week. As food and water consumption on TSOD-E and TSOD-C during the experimental period were almost the same (data not shown), those differences were not due to the energy intake but the $\beta$-CRX administration.

As $\beta$-CRX consumption did not affect the body weight of nonobese mice, TSNO, it was suggested that $\beta$-CRX may contribute to body weight reduction only in obese individuals.

\section{SERUM LIPIDS}

The serum concentrations of TC, NEFA, and TG were assayed and the results are shown in Figure 2.

There were no differences between TSNO-E and TSNO-C in all three serum lipid concentrations and they significantly elevated in TSOD-C. $\beta$-CRX administration on TSOD resulted in the repression of the serum lipid elevation. TG and NEFA concentrations in TSOD-E declined significantly compared to TSOD-C and repressed to the same level as non-obese control, TSNOE. Although TC concentration in TSOD-E was also significantly repressed compared to TSOD-C, it was still significantly higher than TSNO-E.

$\beta$-CRX administration significantly repressed the elevation of serum lipid concentrations in obese mice, TSOD, while it did not affect the normal mice, TSNO. These results indicate $\beta$-CRX administration normalizes serum lipid rather than repressing their elevation.

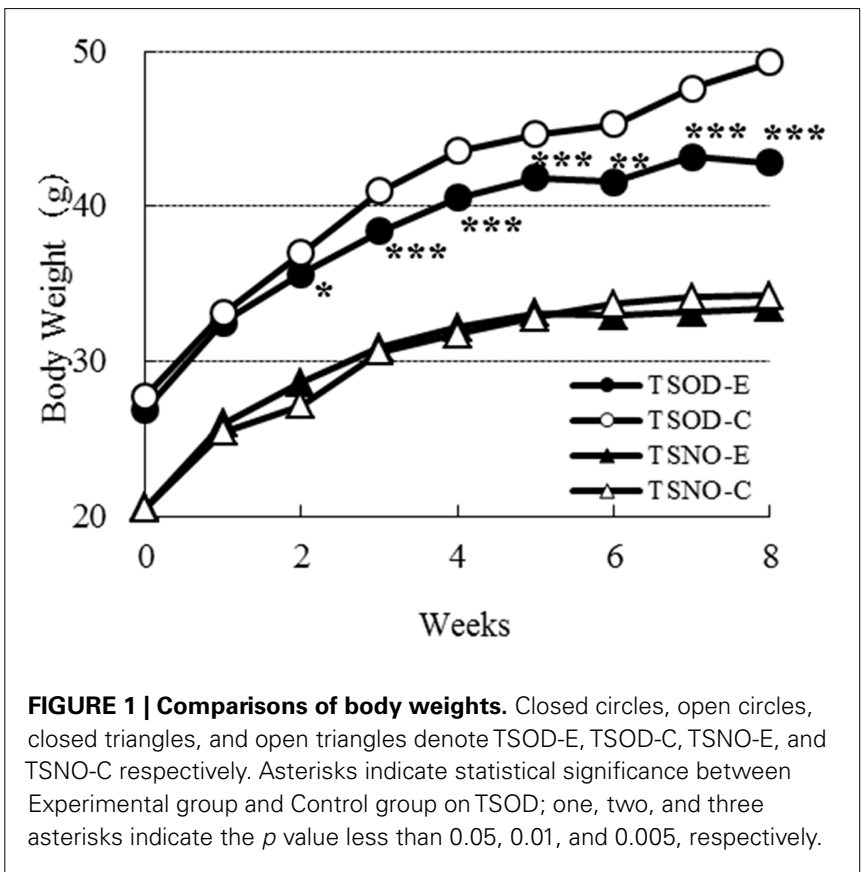




\section{LIVER AND ADIPOSE TISSUES ANALYSIS}

Liver weights were compared between the groups (Figure 3 ). There were no difference between TSNO-E and TSNO-C, while the weight of TSOD-C was significantly higher than those of TSNOs. Though the weight of TSOD-E was still higher than those of TSNOs, it was significantly reduced compared to TSOD-C. Those indicate obese-induced liver hypertrophy was repressed by the administration of $\beta$-CRX.

Similar tendency was observed in three adipose tissue weights; epididymal, perirenal and mesenteric adipose tissues (Figure 4). Each adipose tissue weight in TSOD-E reduced to 86, 80, and $74 \%$ respectively compared to TSOD-C and the total weight reduced to $81 \%$. The weight reduction of mesenteric adipose tissue, commonly called intraperitoneal adipose tissue, was the greatest among them.

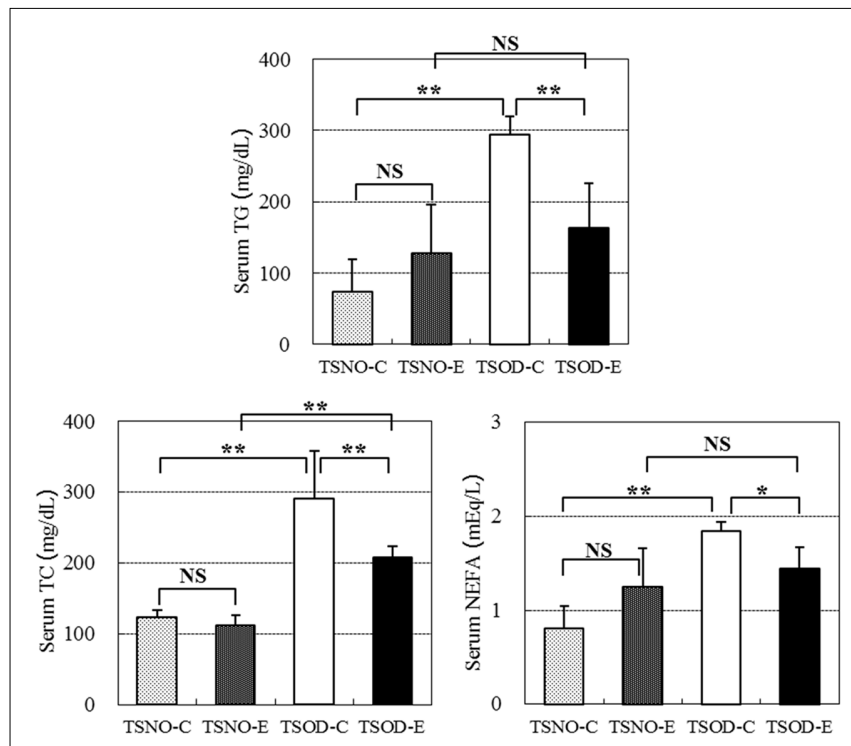

FIGURE 2 | Comparisons of serum lipids. Serum TG, TC, and NEFA concentrations were assayed. One and two asterisks indicate the $p$ value less than 0.05 and 0.01 , respectively and NS indicate no significance between the groups.

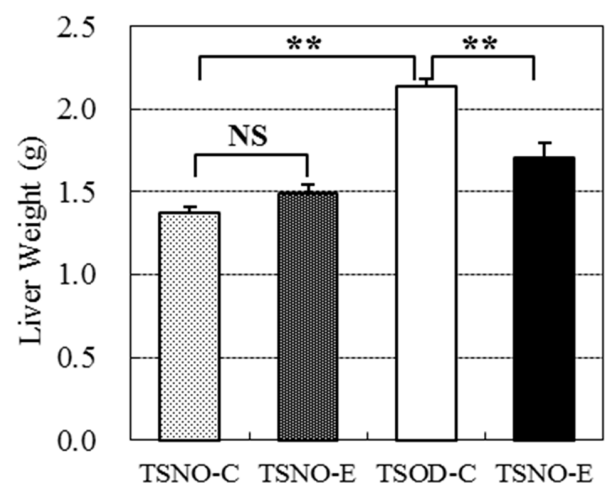

FIGURE 3 | Comparisons of liver weights. Two asterisks indicate the $p$ value less than 0.01 and NS indicate no significance between the groups.
Histocytological microscopic specimens were prepared from epididymal adipose tissues. Figure 5 shows the microscopic images of each adipocyte. The size of adipocyte on TSNO-E and TSNO$\mathrm{C}$ looked almost the same. Although it enlarged significantly on TSOD-C, it reduced on TSOD-E.

The average areas of each adipocyte were calculated to evaluate them objectively (Figure 6). The average areas of TSNO-E and TSNO-C were not different, while it was significantly enlarged on TSOD-C. Compared to TSOD-C, it was significantly reduced on TSOD-E. These observations showed mature adipocytes on TSOD were hypertrophic compared to those on TSNO. But the administration of $\beta$-CRX may prevent adipocyte hypertrophy and normalize them.

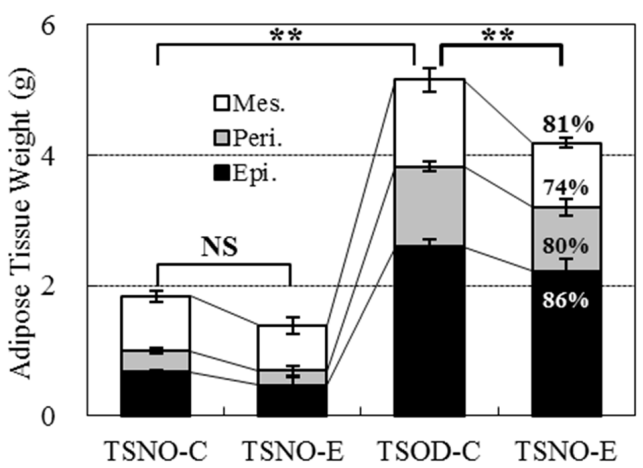

FIGURE 4 | Weights of visceral adipose tissues. Three visceral adipose tissues, epididymal (Epi.), perirenal (Peri.), and mesenteric (Mes.) adipose tissues, were removed and the weights were compared. Percentages in the column of TSOD-E represent the rate of weight reduction compared to TSOD-C. Statistic difference was analyzed against total weight of three tissues; two asterisks indicate the $p$ value less than 0.01 and NS indicates no significance between the groups.
FIGURE 5 | Size of each adipocyte. (A-D) represent HE-stained epididymal adipose tissue section derived from TSNO-C, TSNO-E, TSOD-C, and TSOD-E respectively. 


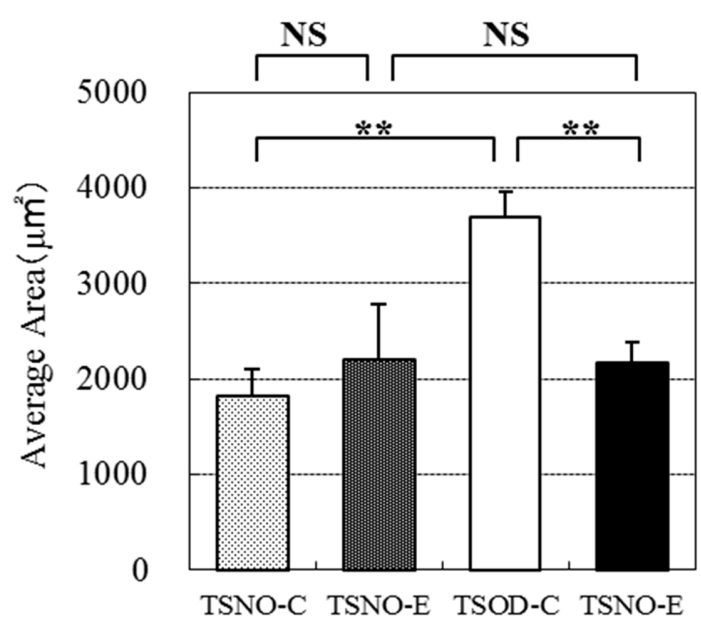

FIGURE 6 | Comparisons of individual adipose area. Average areas of each epididymal cell were calculated and compared. Two asterisks indicate the $p$ value less than 0.01 and NS indicate no significance between the groups.

\section{MICROARRAY ANALYSIS}

The effect of $\beta$-CRX on gene expression on TSOD mouse was evaluated by Affymetrix microarray analysis (Table 1). The number of genes that the expression fluctuating more than two-times both between the TSOD-C and TSNO-C, and between the TSOD-C and TSOD-E on liver, muscle and adipocyte were 270, 56, and 217 respectively.

As the results of functional annotation analysis, lipid metabolism, and transfer were normalized and DNA replication, the first step of cell proliferation, was repressed in the liver on TSODE. In muscle, the repression of the wound response and lipid synthesis and the enhancement of muscle system process were observed. In adipocytes, cell cycle, inflammatory response, and immune system development were repressed. These results indicate $\beta$-CRX facilitates lipid metabolism in muscle and reduced adipocyte proliferation and inflammatory response.

The expression of PPAR- $\alpha$ were also analyzed (Table 2) and found the expression reduction on TSOD-C compared to TSNO$\mathrm{C}$ and it restored on TSOD-E in adipocyte and muscle. In contrast, $P P A R-\alpha$ expression in liver on TSOD-C is almost the same as TSNO-C, while it decreased significantly on TSOD-E compared to TSOD-C. These results indicate oral administration of $\beta$-CRX could modulate the expression of PPAR- $\alpha$. As PPAR- $\alpha$ takes part in many metabolic pathways including energy metabolism, its expression modulation could affects diverse biological processes. The resulting changes might act to prevent obese both directly and indirectly.

\section{DISCUSSION}

Obesity is closely associated with several chronic diseases such as diabetes, cardiovascular diseases, hyperlipidemia, or hypertension, well-known symptoms as metabolic syndrome. As metabolic syndrome is becoming a serious social problem, many efforts have been done to reduce it.
Table 1 | Results of DNA microarray analysis on liver, muscle, and adipocyte.

\begin{tabular}{|c|c|c|c|}
\hline & $\begin{array}{l}\text { Number* } \\
\text { of probes }\end{array}$ & GO term & Regulation \\
\hline \multirow[t]{3}{*}{ Liver } & 270 & $\begin{array}{l}\text { Cholesterol metabolic } \\
\text { process }\end{array}$ & Up/down ${ }^{* *}$ \\
\hline & & Fatty acid metabolism & Up/down ${ }^{* *}$ \\
\hline & & DNA replication & Down \\
\hline \multirow[t]{3}{*}{ Muscle } & 56 & Response to wounding & Down \\
\hline & & Cellular lipid synthesis & Down \\
\hline & & Muscle system process & Up \\
\hline \multirow[t]{2}{*}{ Adipocyte } & 217 & Cell cycle & Down \\
\hline & & Inflammatory response & Down \\
\hline
\end{tabular}

"Number of probes fluctuates the expression > 2-times both between TSNOC/TSOD-C and between TSOD-C/TSOD-E.

${ }^{* *}$ Abnormality of gene expressions was normalized.

Table 2 | Fluctuation of PPAR- $\alpha$ expression.

\begin{tabular}{lll}
\hline & TSOD-C/TSNO-C & TSOD-E/TSOD-C \\
\hline Liver & 0.99 & 0.55 \\
Adipocyte & 0.27 & 2.13 \\
Muscle & 0.32 & 1.78
\end{tabular}

We had reported $\beta$-CRX administration on mildly obese male results in visceral fat reduction (Tsuchida et al., 2008). In spite of the clear results, its mechanism was not examined. Consistent with our previous human study, present report showed $\beta$-CRX administration repressed the elevation of body weight, serum lipid levels, and adipose tissue weight on TSOD mice. This indicates $\beta$-CRX administration could prevent metabolic syndrome both on mouse and human.

To identify the anti-obese mechanism, we focused on the visceral adipose tissue first. Adipose tissue has two functions: energy storage and active endocrine organ and these are closely related. Mature adipocyte accumulates adequate amount of triglyceride and secrete several adipokines such as adiponectin or leptin. Obesity leads over-accumulation of triglyceride to the adipocyte and the subsequent hypertrophic adipocytes secrete inflammatory messengers such as TNF- $\alpha$, IL1- $\beta$, or IL-10 instead of the beneficial ones (Rabe et al., 2008). This plays a role in insulin resistance and deteriorates diabetes or adiposity.

DNA microarray analysis indicates oral administration of $\beta$ CRX could repress the adipocyte proliferation and the inflammatory responses by the inhibition of inflammatory cytokine secretion. These results are well consistent with the present microscopic observation.

PPAR is a nuclear receptor identified in Xenopus (Issemann and Green, 1990). Close relation were known between the upregulation of PPARs and obese or diabetes and they are practically used as the drug; PPAR- $\gamma$ agonists are used for diabetes and PPAR- $\alpha$ agonists are used for serum lipids reduction. 
We recently reported $\beta$-CRX could negatively modulate PPAR- $\gamma$ activity in vitro, not by the direct interaction but though the signaling cascade (submitted). In this study, we showed oral administration of $\beta$-CRX on TSOD mice upregulated PPAR- $\alpha$ expression in adipocyte and muscle, indicating the energy consumption elevation. As $\beta$-CRX also downregulate PPAR- $\alpha$ in the liver of TSOD-E, it is the opposite change found in obese model mice (Memon et al., 2000) and supposed to be an anti-obese effect.

PPAR- $\alpha$ upregulation could increase energy consumption and that contributes to the obese prevention. Because PPAR- $\alpha$ is a nuclear receptor that could affect many cellular and metabolic processes, it might be possible that PPAR- $\alpha$ could contribute to obese prevention though the different pathway. One of the candidates is clock gene-dependent pathway.

We identified circadian rhythm-related gene expression and found interesting changes. For example, Bmall expression in adipocyte increased 3.3 times on TSOD-C compared to TSNO$\mathrm{C}$, while it decreased to 0.6 time by the $\beta$-CRX administration (data not shown).

Several reports indicate the relation between the circadian gene regulation, obese and PPARs. For example, Oishi et al. (2005) showed that PPAR- $\alpha$ agonist could modulate mouse circadian rhythm. Inoue et al. (2005) reported CLOCK/BMAL1 heterodimer involves in lipid metabolism via transcriptional activation of PPAR. Another report showed the crosstalk between

\section{REFERENCES}

Bramlev, P. V. (2000). Is lycopene beneficial to human health? Phytochemistry 54, 233-236.

Cooney, R. V., Bertram, J. S., Hankin, J. H., Kolonel, L. N., Miyake, A., Billings, K., and Bourne, W. (1991). Relationship between dietary, serum, and tissue levels of carotenoids. Cancer Lett. 61, 81-87.

Glise, D., Riondel, J., and Favier, A. (1998). Comparative distribution of $B$-carotene and lycopene after intraperitoneal administration in mice. In vivo 12, 447-454.

Hirayama, I., Yi, Z., Izumi, S., Arai, I., Suzuki, W., Nagamachi, Y., Kuwano, H., Takeuchi, T., and Izumi, T. (1999). Genetic analysis of obese diabetes in the TSOD mouse. Diabetes 48, 1183-1191.

Huang, D. W., Sherman, B. T., and Lempicki, R. A. (2009). Systematic and integrative analysis of large gene lists using DAVID bioinformatics resources. Nat. Protoc. 4, 44-57.

Inoue, I., Shinoda, Y., Ikeda, M., Hayashi, K., Kanazawa, K., Nomura, M., Matsunaga, T., Xu, H., Kawai, S., Awata, T., Komada, T., and Katayama, S. (2005). CLOCK/BMAL1 is involved in lipid metabolism via Transactivation of the peroxisome proliferatoractivated receptor (PPAR) response element. J. Atheroscler. Thromb. 12, 169-174.
Issemann, I., and Green, S. (1990). Activation of a member of the steroid hormone receptor superfamily by peroxisome proliferators. Nature 18 , 645-650.

Kanehisa, M. (1997). A database for post-genome analysis. Trends Genet. 13, 375-376.

Kaplan, L. A., Lau, J. M., and Stein, E. A. (1990). Carotenoid composition, concentration, and relationships in various human organs. Clin. Physiol. Biochem. 8, 1-10.

Mangels, A. R., Holden, J. M., Beecher, G. R., Forman, M. R., and Lanza, E. (1993). Carotenoid content of fruits and vegetables: an evaluation of analytic data. J. Am. Diet. Assoc. 93, 284-296.

Memon, R. A., Tecott, L. H., Nonogaki, K., Beigneux, A., Moser, A. H., Grunfeld, C., and Feingold, K. R. (2000). Up-regulation of peroxtors (PPAR- $\alpha$ ) and PPAR- $\gamma$ messenger ribonucleic acid expression in the liver in murine obesity: troglitazone induces expression of PPAR- $\gamma$-responsive adipose tissuespecific genes in the liver of obese diabetic mice. Endocrinology 141, 4021-4031.

Nair, P. P., Lohani, A., Norkus, E. P., feagins, H., and Bhagavan, H. N. (1996). Uptake and distribution of carotenoids, retinal, and tocopherols in human colonic epithelial cells isome proliferator-activated recep-

CLOCK/BMAL1-regulated and PPARs/RXR $\alpha$-regulated systems (Nakamura et al., 2008).

These results suggest the close relation between the circadian system and the lipid metabolism. Because $\beta$-CRX could modulate PPAR- $\alpha$ gene expression, it could modulate not only the lipid metabolism but also the circadian clock gene expression. In this study we examined the circadian clock related gene expression only one time point a day. Further investigation, e.g., time-dependent gene expression surveys or ethological analysis, may reveal new insight into the relation between circadian regulation and lipid metabolism or metabolic syndrome.

In this study, we demonstrated oral administration of $\beta$-CRX repressed body weight and adipocyte hypertrophy. They may prevent reduction of adipokines and escalation of inflammatory messengers. Serum lipid repression had also observed and was well correspond to the microarray analysis. These results strongly suggest $\beta$-CRX might modulate gene expression, probably via PPAR signaling pathways. Recent findings indicate the involvement of the circadian clock genes in fatty acid metabolism or adiposity (Inoue et al., 2005; Oishi et al., 2005). Although we only analyzed circadian gene expression at one time point a day, $\beta$-CRX could modulate or normalize them through the regulation of PPARs and result in obese prevention. Detailed research may reveal the correlation between obesity and circadian control.

in vivo. Cancer Epidemiol. Biomarkers Prev. 5, 913-916.

Nakamura, K., Inoue, I., Takahashi, S., Komoda, T., and Katayama, S. (2008). Cryptochrome and period proteins are regulated by the CLOCK/BMAL1 gene: crosstalk between the PPARs/RXR $\alpha$-regulated and CLOCK/BMAL1-regulated systems. PPAR Res. 348610, 1155-1164.

Nishino, H., Tokuda, H., Murakoshi, M., Satomi, Y., Masuda, M., Onozuka, M., Yamaguchi, S., Takayasu, J., Tsuruta, J., Okuda, M., Khachik, F., Narisawa, T., Takastuka, N., and Yano, M. (2000). Cancer prevention by natural carotenoids. Biofactors 13, 89-94.

Oishi, K., Shirai, H., and Ishida, N. (2005). CLOCK is involved in the circadian transactivation of peroxisome-proliferatoractivated receptor $\alpha$ (PPAR- $\alpha)$ in mice. Biochem. J. 386 , 575-581.

Rabe, K., Lehrke, M., Parhofer, K. G., and Broedl, U. C. (2008). Adipokines and insulin resistance. Mol. Med. 14, 741-751.

Rauscher, R., Edenharfer, R., and Platt, K. L. (1998). In vitro antimutagenic and in vivo anticlasogenic effects of carotenoids and solvent extracts from fruits and vegetables rich in carotenoids. Mutat. Res. 413, 129-142.
Smith, T. A. (1998). Carotenoids and cancer: prevention and potential therapy. Br. J. Biomed. Sci. 55, 268-275.

Sugiura, M., Kato, M., Matsumoto, H., Nagao, A., and Yano, M. (2002). Serum concentration of B-cryptoxanthin in Japan reflects the frequency of Satsuma mandarin (Citrus unshiu Marc.) consumption. J. Health Sci. 48, 350-353.

Sugiura, M., Matsumoto, H., Kato, M., Ikoma, Y., Yano, M., and Nagao, A. (2004a). Multiple linear regression analysis of the seasonal changes in the serum concentration of B-cryptoxanthin. J. Nutr. Sci. Vitaminol. (Tokyo) 50, 196-202.

Sugiura, M., Matsumoto, H., Kato, M., Ikoma, Y., Yano, M., and Nagao, A. (2004b). Seasonal changes in the relationship between serum concentration of beta-cryptoxanthin and serum lipid levels. J. Nutr. Sci. Vitaminol. (Tokyo) 50, 410-415.

Sugiura, M., Nakamura, M., Ikoma, Y., Yano, M., Ogawa, K., Matsumoto, H., Kato, M., Ohshima, M., and Nagao, A. (2005). High serum carotenids are inversely associated with serum gamma-glutamyltransferase in alcohol drinkers within normal liver function. J. Epidemiol. 15, 180-186. 
Sugiura, M., Nakamura, M., Ikoma, Y., Yano, M., Ogawa, K., Matsumoto, H., Kato, M., Ohshima, M., and Nagao, A. (2006a). Serum carotenoid concentrations are inversely associated with serum aminotransferases in hyperglycemic subjects. Diabetes Res. Clin. Pract. 71, 82-91.

Sugiura, M., Nakamura, M., Ikoma, Y., Yano, M., Ogawa, K., Matsumoto, H., Kato, M., Ohshima, M., and Nagao, A. (2006b). The homeostasis model assessment-insulin resistance index is inversely associated with serum carotenoids in nondiabetic subjects. J. Epidemiol. 16, 71-718.

Sugiura, M., Ogawa, K., and Yano, M. (2006c). Effect of chronic administration of fruit extract (Citrus unshiu Marc.) on glucose tolerance in GK rats, a model of type 2 diabetes. Biosci. Biotechnol. Biochem. 70 293-295.
Sugiura, M., Nakamura, M., Ogawa, K., Ikoma, Y., Ando, F., Shimokata, H., and Yano, M. (2011). Dietary patterns of antioxidant vitamin and carotenoid intake associated with bone mineral density: findings from post-menopausal Japanese female subjects. Osteoporos. Int. 22, 143-152.

Sugiura, M., Nakamura, M., Ogawa, K., Ikoma, Y., Ando, F., and Yano, M. (2008). Bone mineral density in post-menopausal female subjects is associated with serum antioxidant carotenoids. Osteoporos. Int. 19, 211-219.

Suzuki, W., Iizuka, S., Tabuchi, M., Funo, S., Yanagisawa, T., Kimura, M., Sato, T., Endo, T., and Kawamura, H. (1999). A new mouse model of spontaneous diabetes derived from ddY strain. Exp. Anim. 48, 181-189.

Tanaka, T., Kohno, H., Murakami, M., Shimada, R.,
Kagami, S., Sumida, T., Azuma, Y., and Ogawa, H. (2000). Suppression of azoxymethane-induced colon carcinogenesis in male F344 rats my mandarin juices rich in beta-cryptoxanthin and hesperidin. Int. J. Cancer 88, 146-150.

Tsuchida, T, Mukai, K., Mizuno, Y. Masuko, K., and Minagawa, K. (2008). The comparative study of ß-cryptoxanthin derived from Satsuma mandarin for fat of human body. Jpn. Pharmacol. Ther. 36, 247-253.

Uchiyama, S., and Yamaguchi, M. (2006). Oral administration of betacryptoxanthin prevents bone loss in ovariectomized rats. Int. J. Mol. Med. 17, 15-20.

Young, A. J., and Lowe, G. M. (2001). Antioxidant and prooxidant properties of carotenoids. Arch. Biochem. Biophys. 385 , 20-27.
Conflict of Interest Statement: The author declares that the research was conducted in the absence of any commercial or financial relationships that could be construed as a potential conflict of interest.

Received: 29 August 2011; accepted: 11 October 2011; published online: 23 November 2011.

Citation: Takayanagi K (2011) Prevention of adiposity by the oral administration of $\beta$-cryptoxanthin. Front. Neur. 2:67. doi: 10.3389/fneur.2011.00067 This article was submitted to Frontiers in Sleep and Chronobiology, a specialty of Frontiers in Neurology.

Copyright (C) 2011 Takayanagi. This is an open-access article subject to a nonexclusive license between the authors and Frontiers Media SA, which permits use, distribution and reproduction in other forums, provided the original authors and source are credited and other Frontiers conditions are complied with. 